\title{
Finite-temperature investigation of quarter filled ladder systems
}

\author{
C. Gabriel ${ }^{\text {a }}$, E. Sherman ${ }^{\text {a }}$, T. C. Lang ${ }^{\text {a }}$, M. Aichhorn ${ }^{\text {a }}$, H. G. Evertz ${ }^{\text {a,* }}$ \\ ${ }^{\mathrm{a}}$ Institute for Theoretical and Computational Physics, Graz University of Technology, Austria
}

\begin{abstract}
We investigate charge ordering in a quarter-filled ladder at finite temperature by determinantal Quantum Monte Carlo. The sign problem is moderate in a wide range of model parameters relevant for $\mathrm{NaV}_{2} \mathrm{O}_{5}$. The charge order parameter exhibits a crossover as a function of inverse temperature $\beta$ on finite systems. Above a critical nearest neighbor Coulomb repulsion $V_{\mathrm{c}}$, the correlation length grows exponentially with $\beta$, indicative of the ordered phase at $\beta=\infty$. We find a clear single-particle gap manifesting itself in a flat $n(\mu)$ dependence at large nearest neighbor Coulomb repulsion $V$.
\end{abstract}

Key words:

quarter-filled ladders, charge ordering, quantum Monte Carlo

The inorganic ladder compound $\mathrm{NaV}_{2} \mathrm{O}_{5}$ has attracted great attention in recent years. This interest was triggered by magnetic susceptibility measurements [1], which show a phase transition at $T=34 \mathrm{~K}$ into a low-temperature spin-gapped phase. This transition is accompanied by charge ordering, as observed in NMR measurements [2], where the valence of the vanadium sites changes from $\mathrm{V}^{4.5}$ to $\mathrm{V}^{4.5 \pm \delta}$, with $\delta$ the amount of charge disproportion. This transition has been studied theoretically by several techniques at $T=0$ [3].

On a microscopic level the system can be described by an extended Hubbard model

$$
\begin{aligned}
H= & -\sum_{\langle i j\rangle, \sigma} t_{i j}\left(c_{i \sigma}^{\dagger} c_{j \sigma}+\text { H.c. }\right)+U \sum_{i} n_{i \uparrow} n_{i \downarrow} \\
& +V \sum_{\langle i j\rangle} n_{i} n_{j}-\mu \sum_{i} n_{i},
\end{aligned}
$$

at quarter filling $\langle n\rangle=0.5$, with hopping matrix elements $t_{i j}=t_{x}$ along the ladder and $t_{i j}=t_{y}$ within a rung, and chemical potential $\mu$. We state all energies in units of $t_{y}$. These hopping parameters as well as the onsite Coulomb interaction can be extracted from firstprinciple calculations [4]. The hopping along chains

\footnotetext{
* Corresponding author: e-mail: evertz@tugraz.at
}

$t_{x} \simeq 0.5 t_{y}$ is weaker than along rungs. This strongly influences the physics of the ladder, for which a spingap seems to appear at $t_{x} \gtrsim t_{y}[3]$.

We used $t_{x}=0.5$ and $U=8$. Since the non-local Coulomb interaction $V$ cannot be determined properly by band-structure calculations, we used $V$ as a free parameter of the Hamiltonian. The charge order parameter is $\Delta_{\text {co }}^{2}=\frac{1}{2 L\langle n\rangle} \sum_{i j} e^{\mathrm{i} \mathbf{Q}\left(\mathbf{r}_{i}-\mathbf{r}_{j}\right)}\left(n_{i}-\langle n\rangle\right)\left(n_{j}-\langle n\rangle\right)$ with $\mathbf{Q}=(\pi, \pi)$, which is unity for complete ordering.

We performed grand canonical calculations by determinantal quantum Monte Carlo. These are often very difficult for doped systems because of a sign problem. Fortunately, the average sign is favorably large in the relevant parameter range of $t_{x} / t_{y}=0.5$ and large $V$ (Fig. 1). In the opposite case of isotropic $t_{x}=t_{y}$ at small $V$, $\langle\operatorname{sign}\rangle$ becomes very small. The charge order parameter exhibits similar behavior, but it is less strongly dependent on $t_{x} / t_{y}$. Charge order grows with increasing $V$.

Fig. 2 shows the charge correlation length $\xi_{\text {cc }}$. At small interactions, $V=1.5$ and 2.0 , the correlation length seems to saturate, but for $V=2.5$ and 3.0 it increases exponentially with $\beta$, with a $V$-dependent slope. This behavior is consistent with the 1D Ising model in a transverse field (IMTF) [5], which is equiv- 


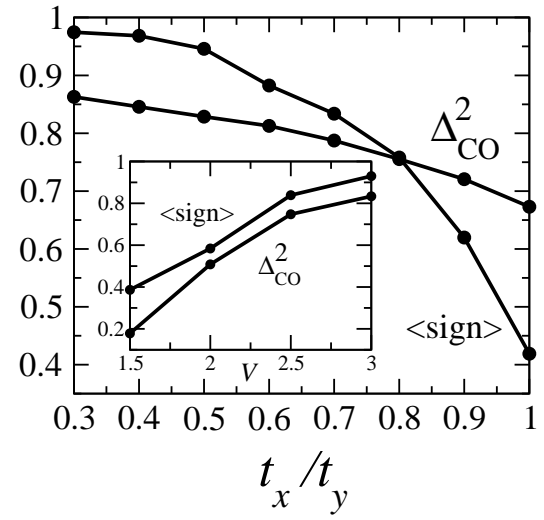

Fig. 1. Mean value of sign and order parameter $\Delta_{\text {co }}^{2}$ as functions of $t_{x} / t_{y}$ at $V=3, \beta=6, L=16,\langle n\rangle=0.5$, and as functions of $V$ at $\beta=6$ (inset).

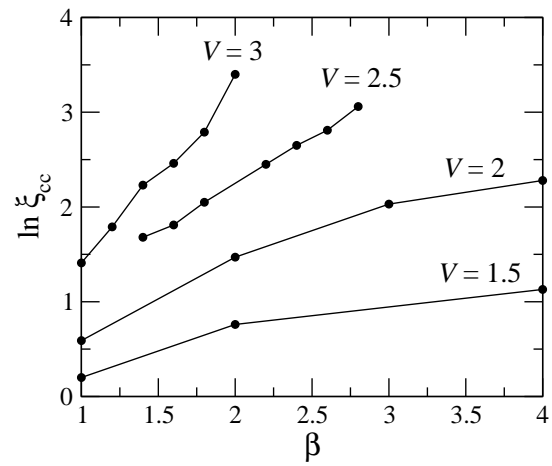

Fig. 2. Logarithm of the charge correlation length $\xi_{\mathrm{cc}}$ as a function of $\beta$ for different interactions $V$ ( $L=32$ for $V=1.5$ and $2 ; L=44$ for $V=2.5$ and 3 )

alent to Eq. 1 in the limit of one spinless electron per rung. For large $V$, the transverse field goes to zero, and $\xi_{\text {IMTF }}=|\ln \tanh (\beta)|^{-1}$. This is exponential behavior with slope 2 at large $\beta$, which the data in Fig. 2 appear to approach. There is long range order in the thermodynamic limit only at $\beta=\infty$. For weaker interactions, $V<2 t_{y}$, the correlation length $\xi_{\text {IMTF }}$ remains finite even in the limit $\beta \rightarrow \infty$, showing a disordered phase at all temperatures. The results in Fig. 2 are nicely consistent with recent DMRG calculations [6] which show that at $T=0$ the system has a quantum phase transition to an ordered phase at $V_{c}=2.1(1)$.

However, the behavior of finite size systems is different from the IMTF in the thermodynamic limit. As a function of inverse temperature, the charge order parameter exhibits a crossover at large $V$ (Fig. 3). For the single ladder, this crossover is a finite size effect. It appears since at some $\beta$, the charge order correlation length will exceed the system size, resulting in apparent long range order. For a three-dimensional system of coupled ladders, this crossover can become a phase transition. The order parameter at $V=3$ and different

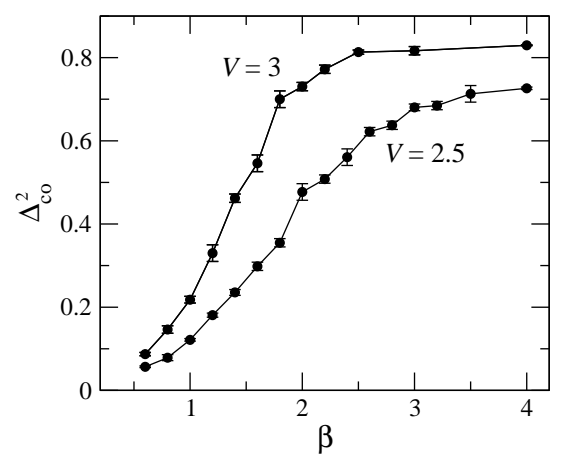

Fig. 3. Charge order parameter as a function of $\beta$ for $V=3.0$ and $V=2.5$ with $L=32$ rungs.

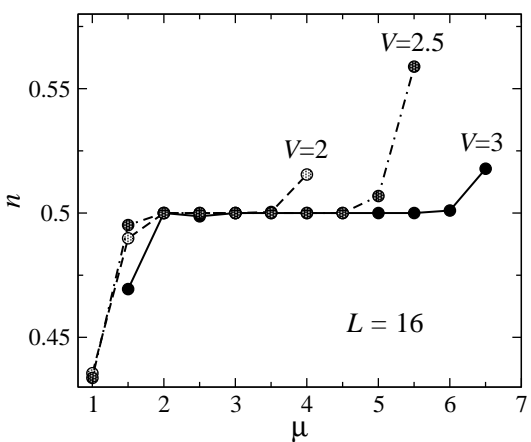

Fig. 4. Mean electron density as a function of the chemical potential $\mu$ at $\beta=6, \mathrm{~L}=16$.

$\beta$ scales well as a function of $\xi(L) / L$.

The onset of charge order at large $V$ is most clearly visible in the single particle gap shown in Fig. 4. It manifests itself as a plateau in the $n(\mu)$ dependence where $\langle n\rangle=0.5$ remains constant in a region $\mu_{\min }<$ $\mu<\mu_{\text {max }}$. At large $V$, the upper boundary $\mu_{\max }$ shifts with $V$ as approximately $3 V$, which is the same value as in the atomic limit at full ordering.

We greatfully acknowledge support by the Austrian Science Fund (FWF), project P15520. M.A. is supported by DOC (Austrian Academy of Sciences).

\section{References}

[1] M. Isobe and Y. Ueda, J. Phys. Soc. Jpn. 65, 1178 (1996).

[2] T. Ohama et al., Phys. Rev. B 59, 3299 (1999).

[3] M. Vojta, A. Hübsch, and R.M. Noack, Phys. Rev. B 63, 045105 (2001). E. Orignac and R. Citro, Eur. Phys. J. B 33, 419 (2003). M. Aichhorn et al., Phys. Rev. B 69, 245108 (2004).

[4] H. Smolinski et al., Phys. Rev. Lett. 80, 5164 (1998). J. Spitaler et al., Physica Scripta T109, 159 (2004) and cond-mat/0404076

[5] E. Lieb, T. Schultz, and D. Mattis, Ann. Phys. 16, 407 (1961). B M. McCoy, Phys. Rev. 173, 531 (1968). 
[6] B. Edegger, R. Noack, and H.G. Evertz, in preparation. 